\title{
Metastability of Band-Gap Energy in GaInNAs Compound Investigated by Photoreflectance
}

\author{
R. Kudrawiec**, J. Misiewicz \\ Institute of Physics, Wrocław University of Technology \\ Wybrzeże Wyspiańskiego 27, 50-370 Wrocław, Poland \\ E.-M. Pavelescu, J. Konttinen and M. Pessa \\ Optoelectronics Research Center, Tampere University of Technology \\ P.P. Box 692, 33101 Tampere, Finland

\begin{abstract}
The band-gap energy of GaInNAs layers lattice-matched to GaAs substrate and annealed under different temperatures is investigated by photoreflectance spectroscopy. Different nitrogen nearest-neighbor environments of $\mathrm{N}$ atom appear in GaInNAs layers due to the post-growth annealing. It leads to an energy-fine structure of the band gap, i.e. well separated photoreflectance resonances related to different nitrogen nearest-neighbor environments $\left(\mathrm{N}-\mathrm{Ga}_{4-m} \operatorname{In}_{m}(0 \leq m \leq 4)\right.$ short-range-order clusters). The temperature dependence of the band gap $E(T)$ related to different $\mathrm{N}-\mathrm{Ga}_{4-m} \mathrm{In}_{m}$ clusters is investigated in $10-280 \mathrm{~K}$ temperature range, and Varshni and Bose-Einstein parameters for $E(T)$ are determined.
\end{abstract}

PACS numbers: 73.22.-f, 78.66.--w, 78.30.--j

\section{Introduction}

Recently, the quaternary GaInNAs compound has attracted a great attention due to its potential application for long wavelength optoelectronic devices on GaAs substrates [1, 2]. So far, many high efficient GaInNAs-based lasers have been reported [3-6]. However, physics of GaInNAs is still not fully understood and has been under intensive study since the last few years (see Refs. [7-18] and

*corresponding author; e-mail: robert.kudrawiec@pwr.wroc.pl 
references therein). Different approaches such as the band anticrossing model, empirical pseudopotential supercell method, first principles pseudopotential method, and tight-binding method were proposed in order to explain the GaInNAs band structure and its optical properties [7-13]. Many of the approaches consider a single structural motif throughout the alloy volume (isomorphous models). Experimental verifications show a significant scatter in results depending on the sample preparation. It indicates that GaInNAs is very metastable compound and an isomorphous model can be valid in some cases because more than one structural motif could be significant in a sample. In exhaustive theoretical treatment the following structural motifs should be taken into consideration: (i) different nitrogen nearest-neighbor environments, (ii) N-related defect states inside the band gap and above the conduction band, and (iii) alloy content fluctuations. Due to the immense complexity of GaInNAs system such extended model seems to be very complicated.

It has been reported many times in the literature that the post-growth annealing strongly influences optical and electrical properties of GaInNAs compounds and QWs (usually leads to an improvement of these properties). Raman scattering [14] and infrared transmission [15-17] studies have shown that the annealing leads to the formation of $\mathrm{N}-\mathrm{In}$ bonds instead of $\mathrm{N}-\mathrm{Ga}$ ones. The change in the nitrogen bonds, thereby in the nitrogen nearest-neighbor environment, strongly influences the band gap of GaInNAs [11]. The reconfiguration of nitrogen surrounding from four $\mathrm{Ga}$ atoms $\left(\mathrm{N}-\mathrm{Ga}_{4}\right)$ to four In atoms $\left(\mathrm{N}-\mathrm{In}_{4}\right)$ leads to an effective blueshift of band-gap energy at the same composition of the compound. This phenomenon has been mainly investigated using photoluminescence spectroscopy which is not sensitive to the possible energy-fine structure of the band gap as photoreflectance (PR) technique. To date, only few works have been devoted to PR investigations of the short-range ordering effect in GaInNAs system [11, 18, 19]. In addition, the temperature dependence of band-gap energy $E(T)$ related to different nitrogen nearest-neighbor environments has never been reported. So far reported investigations into the $E(T)$ relationship for $\mathrm{Ga}(\mathrm{In}) \mathrm{NAs}$ shows that the incorporation of a few percent of $\mathrm{N}$ atoms into host GaAs or InGaAs matrix, besides a redshift of the band-gap energy, leads to a significant decrease in the temperature-induced shrinkage of the band-gap energy, i.e. significantly changes the $E(T)$ relationship [20-24]. The shift of the band-gap energy between 10 and $300 \mathrm{~K}$ for N-diluted $\mathrm{Ga}$ or GaInAs is about $30 \%$ smaller than the shift for $\mathrm{N}$-free compounds. This phenomenon could be understood within BAC model [7], and many authors use this model to explain the N-induced change in the $E(T)$ relationship $[22,23]$.

In this paper we show PR investigations of the band-gap energy for the as-grown and annealed GaInNAs layers in the temperature range of 10-300 K. We have found a set of discrete band gaps related to the different short-range ordering of $\mathrm{N}$ atoms. In addition, different temperature dependences of the particular band-gap energies have been observed. The obtained results are discussed 
in the framework of the tight-binding calculation which explains the strong dependence of the band-gap energy on the nitrogen nearest-neighbor environments, see in Ref. [11]. Also, models considering structural defects are quoted, because it has been observed that this "structural motif" cannot be ignored for as-grown GaInNAs layer. The most interesting phenomenon observed is the coexistence of different band gaps in one sample. This observation confirms cluster-like properties of the metastable GaInNAs system.

\section{Experimental}

The samples discussed in this paper were grown by MBE on $n$-type GaAs:Si (100) substrates at a growth temperature of $470^{\circ} \mathrm{C}$. Each sample consisted of a GaAs buffer layer, a $300 \mathrm{~nm}$ thick $\mathrm{Ga}_{0.942} \mathrm{In}_{0.058} \mathrm{~N}_{0.028} \mathrm{As}_{0.972}$ layer, and a $10 \mathrm{~nm}$ GaAs cap layer, all nominally undoped. The samples were annealed ex situ at 800 and $900^{\circ} \mathrm{C}$ for 60 seconds. X-ray diffraction (XRD) and Raman data confirm that samples selected to this study are GaInNAs layers with the same content and different nitrogen nearest-neighbor environments [19]. These layers are almost lattice matched to GaAs substrate $(\Delta a / a \approx 0.15 \%)$. The environment changes from Ga-rich for the as-grown layer to In-rich for annealed layers [19]. The band-gap energy of GaInNAs layers was investigated by PR spectroscopy. The PR experiments were performed using standard PR apparatus described elsewhere [25]. The samples were mounted on a cold finger in a helium closed cycle refrigerator coupled with a programmable temperature controller allowing measurements in the 10-300 K temperature range.

\section{Results and discussion}

Figure 1a-c shows temperature dependence of $\mathrm{PR}$ spectra recorded for as-grown, annealed at $800^{\circ} \mathrm{C}$, and $900^{\circ} \mathrm{C}$ GaInNAs layers, respectively. It is clearly seen that despite a lack of change in the compound composition PR features related to band-gap absorption shift to blue for annealed layers. Moreover, the line shape of PR resonances changes significantly with the decrease in temperature. It means that PR features associated with new transitions appear at low temperatures. In order to explain these observations a careful analysis of PR data was performed. The PR spectra were analyzed using the standard-critical-point (SCP) model $[26,27]$. According to this model PR spectrum can be fitted using the following formula (i.e. Lorentzian line shape formula):

$$
\frac{\Delta R}{R}=\operatorname{Re}\left[\sum_{j=1}^{n} C_{j} \mathrm{e}^{\mathrm{i} \theta_{j}}\left(\hbar \omega-E_{j}+\mathrm{i} \Gamma_{j}\right)^{-m_{j}}\right],
$$

where $n$ is the number of the spectral functions to be fitted, $\hbar \omega$ is the photon 


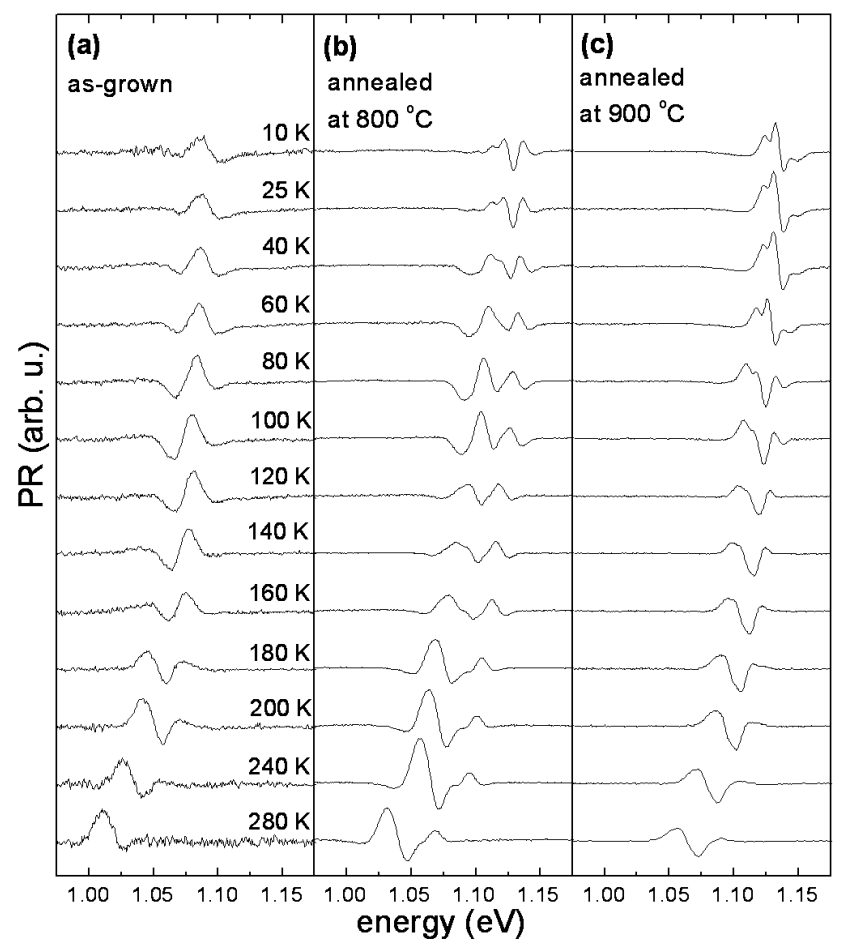

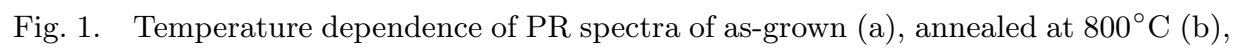
and at $900^{\circ} \mathrm{C}$ (c) $\mathrm{Ga}_{0.942} \mathrm{In}_{0.058} \mathrm{~N}_{0.028} \mathrm{As}_{0.972}$ layers.

energy of the probe beam, $E$ is the CP energy, and $\Gamma, C$, and $\theta$ are the broadening, amplitude, and phase angle, respectively. The term $m$ refers to the type of critical points, i.e. the nature of optical transitions, namely: $m=2.5$ for the three-dimensional one-electron transition.

Figure 2 shows a comparison of PR spectra of the three samples recorded at $280 \mathrm{~K}$. At this temperature an absorption associated with band-to-band transitions is expected. We have observed that experimental data cannot be well reproduced by one PR resonance. Therefore, we assumed that PR spectrum is composed of two resonances. The fitting curves (solid lines) and the modulus of individual PR resonance (dashed lines) are presented together with experimental data in Fig. 2. We have found that the two resonances are separated by not less than $20 \mathrm{meV}$. Such energy difference cannot be attributed to the valence band splitting because our layers are almost lattice-matched to GaAs substrate, i.e. the valence band splitting calculated for these layers with the strain taken after XRD data is less than $6 \mathrm{meV}$. Moreover, the resonances are observed at four discrete energies, i.e. 1.014, 1.036, 1.062, and $1.082 \mathrm{eV}$ (see dashed lines in Fig. 2). It indicates that we do not observe a shift of $\mathrm{PR}$ resonance, but a redistribution of the oscillator strength between a few possible band-to-band transitions. The set of discrete band 


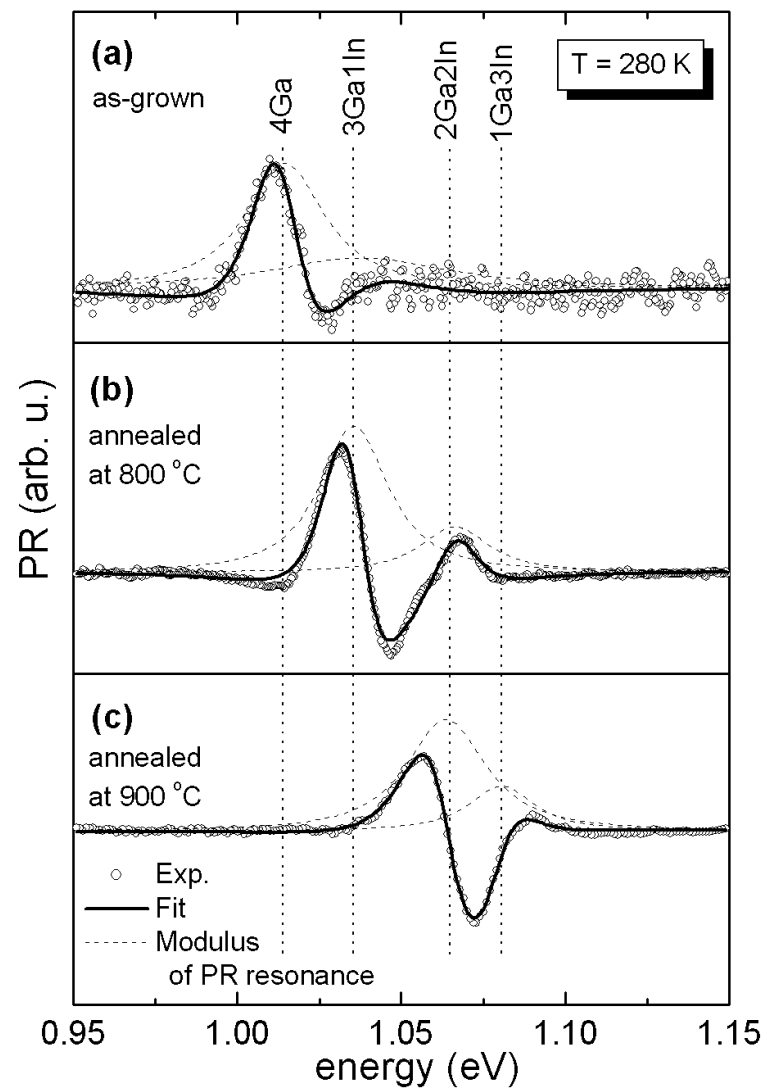

Fig. 2. Comparison of PR spectra of as-grown (a), annealed at $800^{\circ} \mathrm{C} \mathrm{(b),} \mathrm{and} \mathrm{at} 900^{\circ} \mathrm{C}$ (c) $\mathrm{Ga}_{0.942} \mathrm{In}_{0.058} \mathrm{~N}_{0.028} \mathrm{As}_{0.972}$ layers recorded at $280 \mathrm{~K}$ together with fitting curves (solid lines) and the decomposition between the individual modulus of PR resonance (dashed lines).

gaps occurs due to substitution of the isovalent trap, i.e. $\mathrm{N}$ atom, on the sides with different nearest-neighbor environments. During the fast growth of the quaternary layer the $4 \mathrm{Ga}$ configuration, with strongly strained $\mathrm{Ga}-\mathrm{N}$ bonds, is most frequently obtained. However, such bonds configuration is not the most favorable in the terms of crystal energy. The annealing procedure allows a redistribution of atoms in the crystal lattice and the system tends to one with minimal energy, namely the most favorable atom configuration. Therefore, during annealing nitrogen atoms move from Ga-ligand rich sites to In-ligand rich sites without changing the alloy composition. The mechanism of the nitrogen bonds reconfiguration should be a defect-related phenomenon. Therefore, it is expected that this process will be enhanced by As vacancy with In-rich environment.

In the case of as-grown sample, the main PR feature can be interpreted as being due to band-to-band transitions related to the $\mathrm{N}-\mathrm{Ga}_{4}$ configuration (4Ga 
for short). A second feature, located at $25 \mathrm{meV}$ higher energy, can be assigned to the presence of a second nitrogen configuration, such as $3 \mathrm{Ga1} \mathrm{In}$, both co-existing in that particular sample. Upon annealing at $800^{\circ} \mathrm{C}$, the signal arising from the $4 \mathrm{Ga}$ configuration decreases, while that from the $3 \mathrm{Ga1}$ In configuration increases significantly, and a new feature assigned to the $2 \mathrm{Ga} 2 \mathrm{In}$ configuration appears at the high-energy side. Upon annealing at $900^{\circ} \mathrm{C}$, the $3 \mathrm{Ga} 1 \mathrm{In}$ signal disappears and the $2 \mathrm{Ga} 2 \mathrm{In}$ is enhanced. In addition, a feature related to $1 \mathrm{Ga} 3 \mathrm{In}$ configuration can be separated. The last feature could be the most favorable in the term of crystal energy [8], however due to low In concentration this transition cannot be dominant.

With the decrease in temperature PR spectra of as-grown and annealed layers behave differently. In the case of as-grown layers in the whole temperature range $\mathrm{PR}$ spectrum can be fitted by two band-to-band resonances, while in the case of annealed layers new features appear at low temperatures. The new features are related to a splitting of $\mathrm{PR}$ resonance into two components (one related to light hole and second related to heavy hole). Therefore, the low temperature PR spectra of annealed layers should be described by both light-hole and heavy-hole resonances. In the case of as-grown layer the broadening of the individual resonances is so big that these resonances are not resolved in PR spectra.

Figure 3 shows a comparison of PR spectra of the three samples recorded at $10 \mathrm{~K}$. In the case of an as-grown layer two band-to-band resonances reproduce experimental data very well. It confirms the fact that two different nitrogen nearest-neighbor environments (4Ga and $3 \mathrm{Ga} 1 \mathrm{In}$ ) exist in as-grown GaInNAs layer. Also, two different nitrogen environments, i.e. 2Ga2In and $1 \mathrm{Ga} 3 \mathrm{In}$, are dominant for the layer annealed at $900^{\circ} \mathrm{C}$. However, in this case PR spectrum cannot be described by two resonances because such a fit does not reproduce PR data satisfactorily. Therefore, the low-temperature PR spectra recorded for this sample were described by four PR resonances. In the case of GaInNAs layer annealed at $800^{\circ} \mathrm{C} \mathrm{PR}$ features related to three different nitrogen nearest-neighbor environments have been identified. In Fig. 3 we present a fit by two band-to-band and two excitonic resonances in the limited spectral range. Below this spectral range a feature related to $3 \mathrm{Ga1In}$ environment is visible. This feature is very weak in comparison to 2Ga2In and 1 Ga3In ones. However, with the increase in temperature the intensity of this transition increases significantly (see Fig. 1b) and this transition becomes to be dominant at $280 \mathrm{~K}$ (see Fig. 2b). Such decrease in transition intensity with the decrease in temperature is unusual behavior, but it has been previously observed for this compound $[28,29]$. Such behavior of PR signal is associated with the carrier localization phenomenon [28]. For these samples this effect also takes place. With the decrease in temperature we evidently observe a weakness of $4 \mathrm{Ga}$ - and $3 \mathrm{Ga1In}$ related $\mathrm{PR}$ features for the as-grown and annealed at $800^{\circ} \mathrm{C}$ layers, respectively. For example in the case of an as-grown layer, it causes that the 3Ga1In-related resonance is stronger than $4 \mathrm{Ga}$ one at $10 \mathrm{~K}$ (see in Fig. 2a), while at $280 \mathrm{~K}$ the 
relative intensity of these two resonances is in opposite relation (see in Fig. 3a). Similar behavior is observed for the layer annealed at $800^{\circ} \mathrm{C}$. For this sample, the fitting procedure is difficult if contributions related to three different band gaps are comparable (see for example spectra between 60 and $160 \mathrm{~K}$ in Fig. 1b). On the other hand, the multiresonance character of PR spectrum testifies to cluster-like properties of GaInNAs compound, i.e. the local character of the band gap.

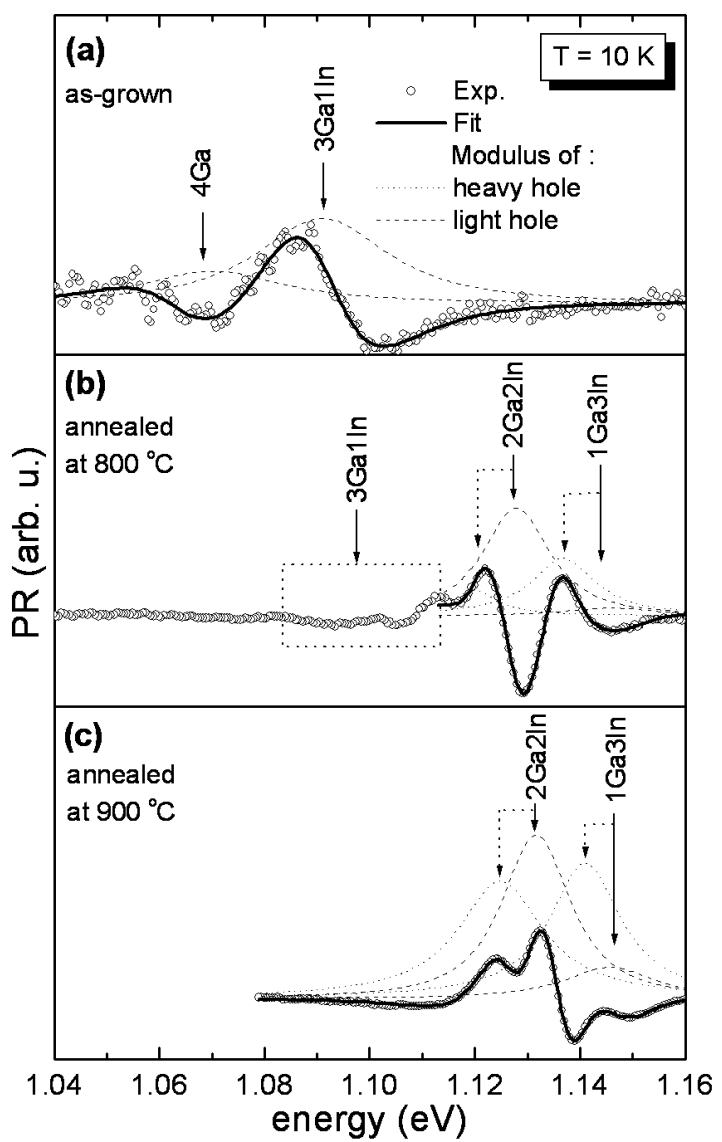

Fig. 3. Comparison of PR spectra of as-grown (a), annealed at $800^{\circ} \mathrm{C}(\mathrm{b})$, and at $900^{\circ} \mathrm{C}$ (c) $\mathrm{Ga}_{0.942} \mathrm{In}_{0.058} \mathrm{~N}_{0.028} \mathrm{As}_{0.972}$ layers recorded at $10 \mathrm{~K}$ together with fitting curves (solid lines) and the decomposition between the individual modulus of PR resonance.

Figure 4 shows energies of excitonic band-to-band transition obtained from the fitting procedure for the as-grown and annealed at $900^{\circ} \mathrm{C}$ layers. Experimental data was fitted using both the Varshni [30] and Bose-Einstein-type [31, 32] (B-E) expressions. The Varshni equation is given by [30]: 


$$
E_{0}(T)=E_{0}(0)-\frac{\alpha T^{2}}{\beta+T},
$$

where $E_{0}(0)$ is the band gap at $T=0 \mathrm{~K}$ while $\alpha$ and $\beta$ are the so-called Varshni coefficients. The B-E expression, which involves electron coupling to an average phonon (optical and acoustical) is given by [31, 32]:

$$
E_{0}(T)=E_{0}(0)-\frac{2 a_{\mathrm{B}}}{\exp \left(\Theta_{\mathrm{B}} / T\right)-1}
$$

where $a_{\mathrm{B}}$ is the strength of the electron-average phonon interaction and $\Theta_{\mathrm{B}}$ is the average phonon temperature.

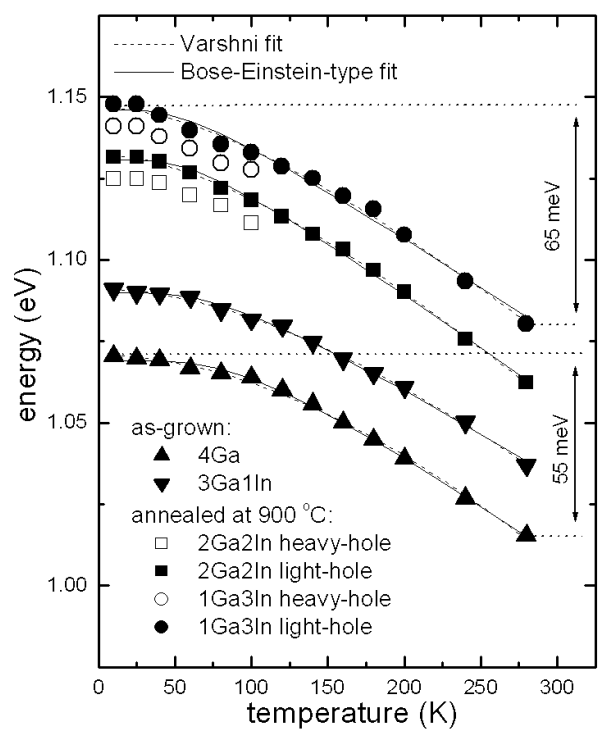

Fig. 4. Temperature dependence of the band-gap energy related to different nitrogen nearest-neighbor environments obtained for as-grown and annealed at $900^{\circ} \mathrm{C}$ $\mathrm{Ga}_{0.942} \mathrm{In}_{0.058} \mathrm{~N}_{0.028} \mathrm{As}_{0.972}$ layers (full points) together with the fit by Varshni (dashed lines) and B-E (solid lines) formulas. The fit-determined parameters are listed in Table.

The dashed and solid lines in Fig. 4 are fits to Eqs. (2) and (3), respectively. The fit-determined parameters, $E_{0}(0), \alpha, \beta, a_{\mathrm{B}}$, and $\Theta_{\mathrm{B}}$, together with literature data [33] are listed in Table. Significant differences in Varshni and B-E parameters are observed for the four different band gaps. Moreover, the literature data is rather poor in this field. It suggests that $E(T)$ relationship depends on the sample preparation and the post-growth treatment. An interpolation of Varshni and B-E parameters for the quaternary GaInNAs compound can be done if we know parameters for the GaN, GaAs, InN, and InAs binary compounds. In order to evaluate these quantities for GaInNAs we can use an interpolation scheme based on the four binaries 


$$
\begin{aligned}
& Q_{\text {quant }}(x, y)=x y Q_{\mathrm{InN}}+x(1-y) Q_{\mathrm{InAs}}+y(1-x) Q_{\mathrm{GaN}} \\
& \quad+(1-x)(1-y) Q_{\mathrm{GaAs}},
\end{aligned}
$$

where $Q_{i}=\alpha_{i}$ or $\beta_{i}(i=$ quant, InN, etc. $) . \alpha, \beta, a_{\mathrm{B}}$, and $\Theta_{\mathrm{D}}$ parameters obtained in this way are close to GaAs parameters due to a small incorporation of In and $\mathrm{N}$ atoms (see in Table). However, experimental values obtained for the as-grown GaInNAs layer are quite far from the calculated parameters. Experimental values tend towards calculated ones for annealed layers, i.e. the band gap related to Inrich environments of $\mathrm{N}$ atoms. This could suggest that the nitrogen surrounding plays an important role in the electron-phonon interaction; thus in the reduction of the temperature dependence of the band-gap energy of GaInNAs. However, a detailed analysis shows that the phenomena related to nitrogen environment are not sufficient to explain the change in Varshni and B-E parameters. The postgrowth annealing, besides changes in nitrogen environment, leads to significant

TABLE

Values of the parameters obtained by fitting to the Varshni and Bose-Einstein-type relation, together with literaturedata. In addition, parameters for $\mathrm{GaN}$, InN, GaAs,

\begin{tabular}{|c|c|c|c|c|c|c|}
\hline Sample & $\begin{array}{c}E_{0}(0) \\
{[\mathrm{eV}]}\end{array}$ & $\begin{array}{c}\alpha \\
{\left[10^{-4}\right.} \\
\mathrm{eV} / \mathrm{K}]\end{array}$ & $\begin{array}{c}\beta \\
{[\mathrm{K}]}\end{array}$ & $\begin{array}{c}E_{0}(0) \\
{[\mathrm{eV}]}\end{array}$ & $\begin{array}{c}a_{\mathrm{B}} \\
{[\mathrm{meV}]}\end{array}$ & $\begin{array}{c}\Theta_{\mathrm{B}} \\
{[\mathrm{K}]}\end{array}$ \\
\hline $\mathrm{GaN}^{a}$ & 3.470 & 6.14 & 870 & 3.470 & 65 & 587 \\
\hline $\operatorname{InN}^{a}$ & 1.994 & 2.2 & 700 & 1.994 & 54 & 561 \\
\hline $\mathrm{GaAs}^{a}$ & 1.519 & 4.76 & 360 & 1.519 & 27 & 251 \\
\hline $\operatorname{In} A s^{a}$ & 0.414 & 2.18 & 262 & 0.414 & 21 & 147 \\
\hline calculated from Eq. (4) & - & 4.7 & 368 & - & 28 & 254 \\
\hline $\begin{array}{c}\mathrm{GaN}_{0.03} \mathrm{As}_{0.97}{ }^{b} \\
\mathrm{Ga}_{0.947} \mathrm{In}_{0.053} \mathrm{~N}_{0.026} \mathrm{As}_{0.974} \\
\text { as-grown }\end{array}$ & 1.154 & 0.61 & 560 & 1.202 & 49 & 285 \\
\hline $4 \mathrm{Ga}$ & $\begin{array}{c}1.0708 \\
\pm 0.0005\end{array}$ & $\begin{array}{c}8.5 \\
\pm 2.7\end{array}$ & $\begin{array}{c}907 \\
\pm 367\end{array}$ & $\begin{array}{c}1.0692 \\
\pm 0.0005\end{array}$ & $\begin{array}{r}49 \\
\pm 5\end{array}$ & $\begin{array}{l}290 \\
\pm 17\end{array}$ \\
\hline $\begin{array}{c}\text { 3Ga1In } \\
\text { annealed at } 900^{\circ} \mathrm{C}\end{array}$ & $\begin{array}{c}1.0910 \\
\pm 0.0004\end{array}$ & $\begin{array}{c}5.4 \\
\pm 0.8\end{array}$ & $\begin{array}{c}504 \\
\pm 105\end{array}$ & $\begin{array}{c}1.0899 \\
\pm 0.0006\end{array}$ & $\begin{array}{l}35 \\
\pm 5\end{array}$ & $\begin{array}{l}240 \\
\pm 19\end{array}$ \\
\hline 2Ga1In & $\begin{array}{c}1.1323 \\
\pm 0.0004\end{array}$ & $\begin{array}{c}4.7 \\
\pm 0.3\end{array}$ & $\begin{array}{l}240 \\
\pm 26\end{array}$ & $\begin{array}{c}1.1309 \\
\pm 0.0007\end{array}$ & $\begin{array}{l}32 \\
\pm 4\end{array}$ & $\begin{array}{l}185 \\
\pm 15\end{array}$ \\
\hline 1Ga3In & $\begin{array}{c}1.1469 \\
\pm 0.0009\end{array}$ & $\begin{array}{c}4.4 \\
\pm 0.7\end{array}$ & $\begin{array}{l}238 \\
\pm 76\end{array}$ & $\begin{array}{c}1.1461 \\
\pm 0.0014\end{array}$ & $\begin{array}{l}26 \\
\pm 7\end{array}$ & $\begin{array}{l}167 \\
\pm 31\end{array}$ \\
\hline
\end{tabular}
InAs, and for $\mathrm{Ga}_{0.942} \mathrm{In}_{0.058} \mathrm{~N}_{0.028} \mathrm{As}_{0.972}$ calculated on the basis of Eq. (4) are listed.

${ }^{a}$ Ref. [33], ${ }^{b}$ Ref. [20] 
reduction in the number of structural defects $[24,34,35]$. The last phenomenon is connected with the carrier localization effect, which is typical of $\mathrm{Ga}$ (In)NAs compounds and can be probed in photoluminescence (PL). So far, many researchers reported PL investigations which support a reduction of defect states [35], therefore a weakness of the carrier localization after annealing [34]. PL spectra, not presented there, show that for our set of GaInNAs layers the annealing process significantly enhances band gap-related emission and decreases defect-related PL band as in Ref. [34]. It confirms the fact that in our samples annealing also leads to a reduction in defect states. Therefore, we can connect the change in Varshni and $\mathrm{B}-\mathrm{E}$ parameters with the two phenomena: the change in nitrogen bonds (i.e. the change in nitrogen nearest-neighbor environment) and the reduction in the number of structural defects. The physical mechanism responsible for the different $E(T)$ relationship is discussed below. We consider only the B-E parameters, because these parameters possess physical interpretation. In the ad hoc Varshni model, the parameter $\alpha$ represents the magnitude of the limiting slope of the corresponding $E(T)$ curve, and $\beta$ is a physically undefinable temperature parameter believed to be of the same order of magnitude as the Debye temperature $\Theta_{\mathrm{D}}$ of the material in question.

We have found that for the as-grown layer the average electron-phonon interaction is about twice stronger than expected. The $a_{\mathrm{B}}$ and $\Theta_{\mathrm{B}}$ parameters decrease after annealing, hence they become more similar to the GaAs parameters. Yaguchi et al. [36] suggest that if electrons are strongly localized at $\mathrm{N}$ atoms they could interact preferentially with the localized $\mathrm{Ga}-\mathrm{N}$ vibration mode with higher frequency mainly due to their localized character. In this case, the $a_{\mathrm{B}}$ and $\Theta_{\mathrm{B}}$ parameters could be a non-linear interpolation between parameters related to GaN, GaAs, InN, and InAs binary compounds. Such a situation seems to be important for our as-grown layer where the number of structural defects is significant and electrons are strongly localized at $\mathrm{N}$ atoms in $4 \mathrm{Ga}$ and $3 \mathrm{Ga1In}$ clusters. Therefore, in our case, the problem is to settle whether the phenomenon of different $E(T)$ relationship is related to the nitrogen nearest-neighbor environment or to defect states.

The equimolar system GaInNAs is to be thought of as GaN + InAs or as GaAs + InN. Material properties for the two extreme situations can be different and can be far from the parameters interpolated using Eq. (4). However, we do not expect a significant difference for the two opposite systems $(\mathrm{GaN}+\mathrm{InAs}$ and $\mathrm{GaAs}+\mathrm{InN}$ ), because GaN and InAs parameters are close to InN and GaAs ones, respectively (see Table). Therefore, we concluded that the annealing induced change in the $E(T)$ relationship should be mainly attributed to the defect states. This conclusion is supported by results shown in Fig. 5. This figure shows the $E(T)$ relationship of $3 \mathrm{Ga} 1 \mathrm{In}$ - and $2 \mathrm{Ga} 2 \mathrm{In}$-band gaps obtained for as-grown and annealed layers. While $E(T)$ dependence of 2 Ga2In band gap is very similar for the layers annealed at 800 and $900^{\circ} \mathrm{C}$, the $E(T)$ relationship of $3 \mathrm{Ga} 1 \mathrm{In}$ band 
gap is different for as-grown and annealed layers. It indicates that the band gap related to the same nitrogen nearest-neighbor environment can have different $E(T)$ relationship. Therefore, we have attributed the variations in $E(T)$ dependence to the presence of defect states at the conduction band. These states are the origin of the tail of the density of state (DOS). If the tail of DOS is comparable for two samples, like for samples annealed at 800 and $900^{\circ} \mathrm{C}$, the $E(T)$ relationship for the two samples should be similar (see Fig. 5). Different $E(T)$ characteristics are expected for samples with different tails of DOS. Therefore, the huge change in $E(T)$ characteristic is observed for the couple of as-grown and annealed layers (see Fig. 5).

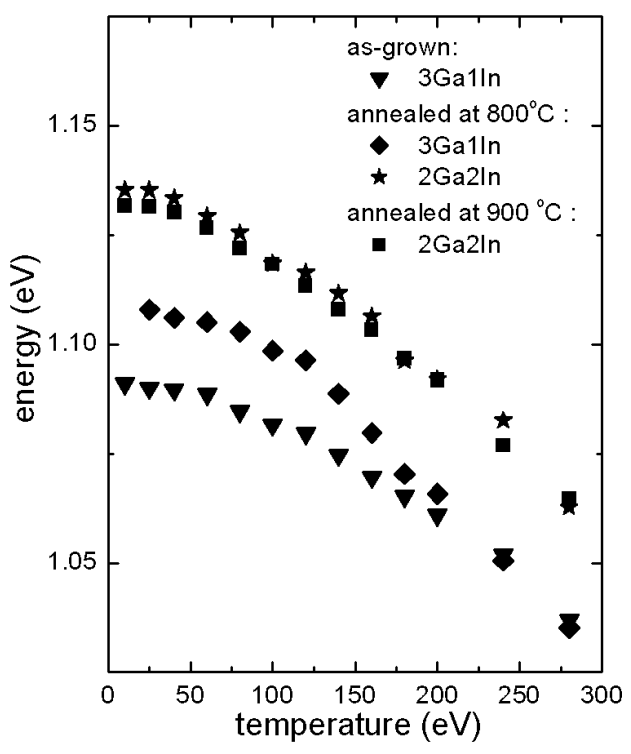

Fig. 5. Comparison of the temperature dependence of the band-gap energy related to 3Ga1In and 2Ga2In nitrogen nearest-neighbor environments for as-grown and annealed at $800^{\circ} \mathrm{C} \mathrm{Ga}_{0.942} \mathrm{In}_{0.058} \mathrm{~N}_{0.028} \mathrm{As}_{0.972}$ layers.

Concerning the interaction of the localized carriers with the local phonon modes with a higher frequency than an average frequency for this system, we have concluded that this phenomenon is important in the limited range of temperatures, i.e. at temperatures for which the strong localization of the carriers takes place. Moreover, such mechanism should be unimportant at low temperatures when the number of phonon modes is low. Therefore, we have estimated that this phenomenon could influence the $E(T)$ relationship in the range of temperatures of $\approx 50-160 \mathrm{~K}$.

If we want to analyse the $E(T)$ relationship, it is important that we have to be certain that investigated transition has the same nature in the whole range of 
temperatures. In the case of the as-grown layer, it is rather difficult to attribute $\mathrm{PR}$ resonance exactly to a pure band-to-band absorption in the whole range of temperatures. For as-grown layers many electron levels at the minimum of conduction band are expected [34]. At low temperatures due to the low concentration of carriers these levels are empty and an extra absorption between the top of valence band and these levels is expected. However, a well defined PR resonance for the extra absorption cannot be expected because these states do not give a well defined critical point in DOS. These states are the origin of the tail of DOS which, as is well known, plays essential role in the absorption at low temperatures. Therefore, at low temperatures the character of the absorption is not pure bandto-band, and the absorption between the top of valence band and the tail of DOS of conduction band influences PR resonance related to the band-to-band absorption. If the temperature rises the carrier concentration rises too, and the tail of DOS becomes unimportant for the absorption process. In the other words, the discrete levels associated with the tail are occupied by electrons and transitions to these levels are impossible. The reduction of the tail of DOS by post-growth annealing leads to a change in the $E(T)$ characteristic. In the case of our samples, we have observed that the temperature-induced band-gap shrinkage between 10 and $280 \mathrm{~K}$ increases from 55 to $65 \mathrm{meV}$ after annealing (see Fig. 4). Figure 4 evidently shows that the $10 \mathrm{meV}$ change in the band-gap shrinkage takes place at low temperatures. Therefore, we have concluded that a significant contribution to

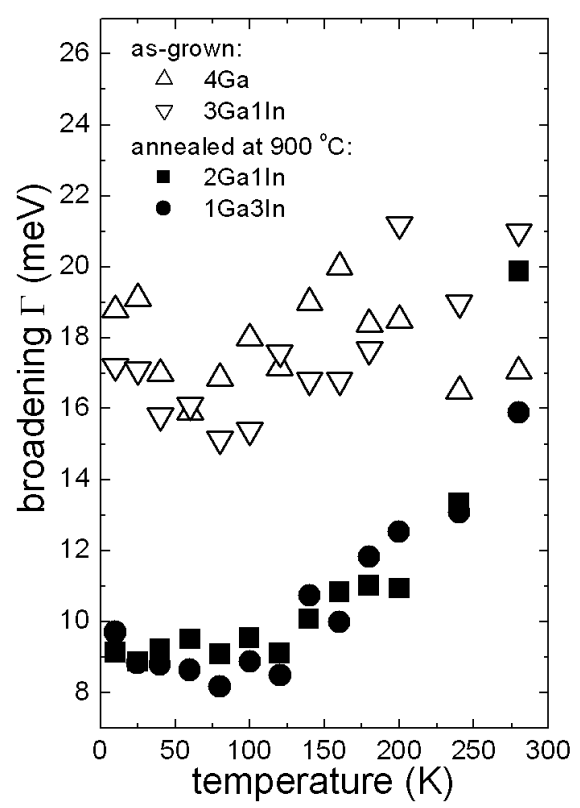

Fig. 6. Temperature dependence of the broadening parameter $\Gamma$ for as-grown (open points) and annealed at $900^{\circ} \mathrm{C}$ (full points) $\mathrm{Ga}_{0.942} \mathrm{In}_{0.058} \mathrm{~N}_{0.028} \mathrm{As}_{0.972}$ layers. 
the temperature-induced band-gap shrinkage derives from the presence of defect states. Post-growth annealing reduces defect states, hence increases the shrinkage of band gap. However, the temperature-induced band gap shrinkage is still much smaller than the shrinkage of host InGaAs matrix (65 vs. $83 \mathrm{meV}$ ).

We also have analyzed the broadening parameter $\Gamma$ of $\mathrm{PR}$ resonance, which is related to the sample quality (see Fig. 6). The temperature dependence of the line width can be expressed as

$$
\Gamma_{0}(T)=\Gamma(0)+\frac{\Gamma_{\mathrm{LO}}}{\exp \left(\Theta_{\mathrm{LO}} / T\right)-1},
$$

where $\Gamma_{0}(0)$ is the broadening mechanism due to intrinsic lifetime, electronelectron interaction, impurity, dislocation, and alloy scattering effects, $\Gamma_{\mathrm{LO}}$ is the electron-longitudinal optical (LO) phonon coupling constant and $\Theta_{\mathrm{LO}}$ is the LO phonon temperature. In the case of as-grown layers, the broadening related to structural defects $\Gamma_{0}(0)$ is about $17 \mathrm{meV}$, hence the contribution of the last term in Eq. (4) cannot be separated. In the case of annealed layers, $\Gamma_{0}(0)$ decreases to $\approx 9 \mathrm{meV}$, hence the increase in the broadening caused by the electron-LO phonon interaction is visible above $140 \mathrm{~K}$. Such behavior of the broadening confirms the fact that the post-growth annealing improves the sample quality.

\section{Conclusions}

In conclusions, we have performed a detailed analysis of the $E(T)$ relationship for the metastable $\mathrm{Ga}_{1-x} \mathrm{In}_{x} \mathrm{~N}_{y} \mathrm{As}_{1-y}$ compound lattice-matched to GaAs substrate. We have observed that for the same composition of the compound, different discrete energies of the band-gap energy occur by rearranging $\mathrm{N}$ environment from Ga-rich to In-rich one. The change in $\mathrm{N}$ environment can be obtained by post-growth annealing and the particular band gaps can be probed by photoreflectance. It has been shown that one of the possible explanations of the phenomena is the presence of five discrete band gaps related to five different nearest-neighbor environments of nitrogen atom. The band gap related to $\mathrm{N}-\mathrm{In}_{4}$ atom configuration was not observed due to too low In concentration. The $E(T)$ relationship for the band gap related to different nitrogen nearest-neighbor environments, i.e. $\mathrm{N}-\mathrm{Ga}_{4-m} \operatorname{In}_{m}(0 \leq m \leq 4)$ short-range-order clusters, has been analyzed. We have concluded that the $E(T)$ relationship is very similar for each $\mathrm{N}-\mathrm{Ga}_{4-m} \operatorname{In}_{m}$ clusters. The other $E(T)$ relationship observed for as-grown layers has been attributed to the presence of defect states. We have shown that both the incorporation of $\mathrm{N}$ atoms in a host GaInAs matrix and the defect states decrease the temperature-induced band-gap shrinkage of the host matrix. We have found that the shift of the band-gap energy between 10 and $280 \mathrm{~K}$ is 55 and $65 \mathrm{meV}$ for as-grown and annealed GaInNAs layers, respectively. It corresponds to the 34 and $22 \%$ decrease in the band-gap shrinkage in comparison to the band-gap shrinkage for GaInAs host matrix. 


\section{Acknowledgments}

This research was supported by the State Committee for Scientific Research (Poland) under grant No. 4 T11B 00823 and grant No. 2P03B 108 25. One of the authors (R. Kudrawiec) acknowledges the financial support from the Foundation for Polish Science.

\section{References}

[1] M. Kondow, K. Uomi, A. Niwa, T. Kikatani, S. Watahiki, Y. Yazawa, Jpn. J. Appl. Phys. 35(2B), 1273 (1996).

[2] J.S. Harris Jr, Semicond. Sci. Technol. 17, 880 (2002).

[3] M. Reinhardt, M. Fischer, M. Kamp, J. Hofmann, A. Forchel, IEEE Photon. Technol. Lett. 12, 239 (2000).

[4] M. Fischer, D. Gollub, A. Forchel, Jpn. J. Appl. Phys. Part 1 41, 1162 (2002).

[5] W. Li, T. Jouhti, C.S. Peng, J. Konttinen, P. Laukkanen, E.-M. Pavelescu, M. Dumitrescu, M. Pessa, Appl. Phys. Lett. 79, 3386 (2001).

[6] M. Kawaguchi, T. Miyamoto, E. Gouardes, D. Schlenker, T. Kondo, F. Koyama, K. Iga, Jpn. J. Appl. Phys. Part 2 40, L744 (2001).

[7] W. Shan, W. Walukiewicz, J.W. Ager III, E.E. Haller, J.F. Geisz, D.J. Friedman, J.M. Olson, S.R. Krutz, Phys. Rev. Lett. 82, 1221 (1999).

[8] P.R.C. Kent, A. Zunger, Phys. Rev. Lett. 86, 2613 (2001).

[9] P.R.C. Kent, A. Zunger, Phys. Rev. B 64, 115208 (2001).

[10] A. Al-Yacoub, L. Bellaiche, Phys. Rev. B 62, 10847 (2000).

[11] P.J. Klar, H. Grüning, J. Koch, S. Schäfer, K. Volz, W. Stolz, W. Heimbrodt, A.M. Kamal Saadi, A. Lindsay, E.P. O'Reilly, Phys. Rev. B 64, 121203(R) (2001)

[12] N.G. Szwacki, P. Boguslawski, Phys. Rev. B 64, 161201 (2001).

[13] I. Gorczyca, C. Skierbiszewski, T. Suski, N.E. Christensen, A. Svane, Phys. Rev. B 66, 081106 (2002).

[14] J. Wagner, T. Geppert, K. Kohler, P. Ganser, N. Herres, J. Appl. Phys. 90, 5027 (2001).

[15] T. Kitatani, M. Kondow, M. Kudo, Jpn. J. Appl. Phys. 40, L750 (2001).

[16] S.R. Kurtz, J.F. Klem, A.A. Allerman, R.M. Sieg, C.H. Seager, E.D. Jones, Appl. Phys. Lett. 80, 1379 (2001).

[17] S. Kurtz, J. Webb, L. Gedvilas, D. Friedman, J. Geisz, J. Olson, R. King, D. Joslin, N. Karam, Appl. Phys. Lett. 78, 748 (2000).

[18] R. Kudrawiec, G. Sek, J. Misiewicz, D. Gollub, A. Forchel, Appl. Phys. Lett. 83, 2772 (2003).

[19] R. Kudrawiec, E.-M. Pavelescu, J. Wagner, G. S/ek, J. Misiewicz, J. Konttinen, M. Pessa, J. Appl. Phys., in press.

[20] K. Uesugi, I. Suemune, T. Hasegawa, T. Akutagawa, T. Nakamura, Appl. Phys. Lett. 76, 1285 (2000). 
[21] I. Suemune, K. Useugi, W. Walukiewicz, Appl. Phys. Lett. 77, 3021 (2000).

[22] R. Chtourou, F. Bousbih, S. Ben Bouzid, F.F. Charfi, J.C. Harmand, G. Ungaro, L. Largeau, Appl. Phys. Lett. 80, 2075 (2002).

[23] A. Nishikawa, R. Katayama, K. Onabe, Y. Shiraki, J. Cryst. Growth 251, 427 (2003).

[24] R. Kudrawiec, G. Sek, J. Misiewicz, L.H. Li, J.C. Harmand, Solid State Commun. 129, 353 (2003).

[25] J. Misiewicz, P. Sitarek, G. Sek, R. Kudrawiec, Mater. Sci. 21, 263 (2003).

[26] D.E. Aspnes, Surf. Sci. 37, 418 (1973).

[27] F.H. Pollak, in: Handbook on Semiconductors, Ed. T.S. Moss, Vol. 2, Elsevier Science, Amsterdam 1994, p. 527.

[28] R. Kudrawiec, J. Misiewicz, L.H. Li, J.C. Harmand, Appl. Phys. Lett. 83, 1379 (2003).

[29] R. Kudrawiec, J. Misiewicz, M. Fischer, A. Forchel, Phys. Status Solidi A 201, 364 (2004).

[30] K.P. Varshni, Physica 34, 149 (1967).

[31] P. Lautenschlager, P.B. Allen, M. Cardona, Phys. Rev. B 33, 5501 (1986).

[32] P. Lautenschlager, M. Garriga, M. Cardona, Phys. Rev. B 35, 9174 (1987).

[33] R. Passler, J. Appl. Phys. 89, 6235 (2001) and references therein.

[34] R. Kudrawiec, G. Sek, J. Misiewicz, L.H. Li, J.C. Harmand, Eur. Phys. J. B, in press.

[35] E.V. Rao, A. Ougazzaden, Y. Le Bellego, M. Juhel, Appl. Phys. Lett. 72, 1409 (1998).

[36] H. Yaguchi, S. Kikuchi, Y. Hijikata, S. Yoshida, D. Aoki, K. Onabe, Phys. Status Solidi B 228, 273 (2001). 